\section{§14. Ion Dynamics in Steady Collisionless Driven Reconnection}

Pei, W.B. (Grad. Univ. Advanced Studies), Horiuchi, R., Sato, T.

In order to investigate a long time scale behavior of collisionless driven reconnection we have developed two-dimensional electromagnetic full particle simulation code for an open system, in which an external driving electric field is assumed at the upstream boundary and free boundary conditions are adopted for the downstream boundary. It is found that a steady regime exists in the temporal evolution of collisionless driven reconnection when a driving flow window is narrow.

The simulation is carried out on a $512 \times 128$ point grid by making use of 6.4 million particles for the input window size $x_{d}=18 \rho_{i}$. Simulation starts from a 1D Harris equilibrium with a characteristic scale $L \approx 3 \rho_{i}$. Figure 1 plots the time history of an electric field at the reconnection point. After overshooting in the early growing phase, the reconnection electric field approaches the external driving field $E_{0}$. Its value remains around the external driving field with a small fluctuation for $t \omega_{c i}>12$. That is, the system relaxes into the steady state in which the reconnection rate is completely controlled by an external driving electric field imposed at the input boundary.

Figure 2 is the time history of five spatial scales in the current sheet. In the early growing phase the width of the current sheet decreases with time and approaches the electron scales. In the steady state, however, both the current density and mass density profiles have the same half-width equal to an ion meandering scale $l_{m i}$. This result indicates that, although the electric current is predominantly carried by electrons, the spatial structure of the current sheet in the steady state is exclusively controlled by the dynamics of meandering ions.

Figure 3 illustrates the spatial structure of ion temperature in the steady state. It is worthy of note that the spatial profile of ion temperature changes from an initial uniform one to a peaked one in the ion current sheet $\left(y<l_{m i}\right)$. The ion heating mechanism is explained in the following. Meandering ions execute an bouncing mo-

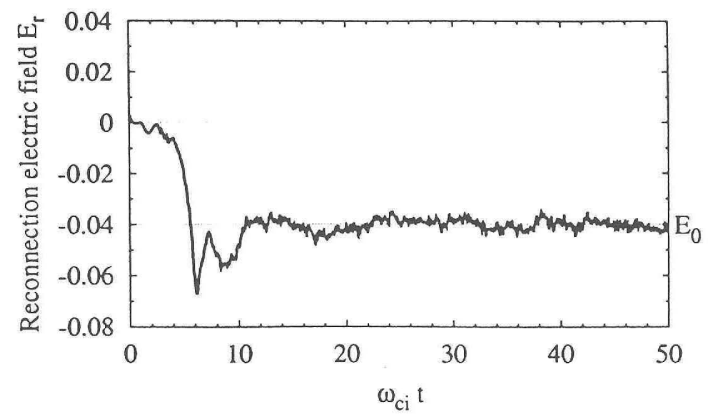

Fig. 1. Temporal evolution of reconnection electric field for $x_{d}=18 \rho_{i}$.

tion across the neutral sheet in the y direction. On the other hand, an electrostatic field is generated through the finite Larmor radius effect in the ion current sheet. Because ions are unmagnetized but electrons remain magnetized, the electrostatic field leads to the acceleration of electrons in the equilibrium current direction through the $\mathbf{E} \times \mathbf{B}$ drift motion and ion heating by intensifying meandering motion in the y direction. Thus the global dynamic behavior of steady reconnection is dominantly controlled by the ion dynamics.

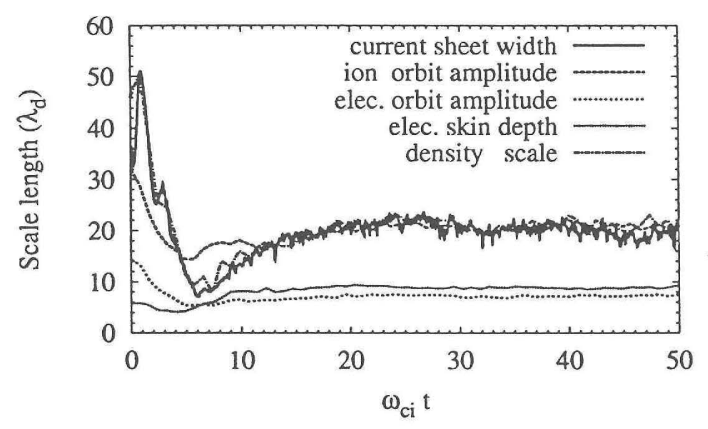

Fig. 2. Temporal evolutions of five spatial scales in the current sheet for $x_{d}=18 \rho_{i}$.

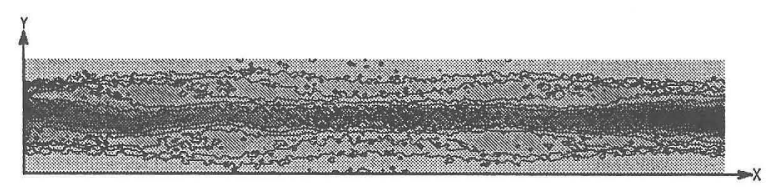

Fig. 3. Spatial structure of ion temperature in the steady state $\left(t \omega_{c i}=49\right)$.

\section{References}

1) R. Horiuchi, W. Pei and T. Sato, Earth, Planets, and Space, in press, 2001.

2) W. Pei, R. Horiuchi and T. Sato, Phys. Plasma, in press, 2001. 